18-fluoro-2-deoxy-D-glucose (FDG) positron emission tomography (PET)/computed tomography $(\mathrm{CT})$ is currently the most valuable imaging technique in Hodgkin lymphoma. Since its first use in lymphomas in the 1990s, it has become the gold standard in the staging and end-of-treatment remission assessment in patients with Hodgkin lymphoma. The possibility of using early (interim) PET during first-line therapy to evaluate chemosensitivity and thus personalize treatment at this stage holds great promise, and much attention is now being directed toward this goal. With high probability, it is believed that in the near future, the result of interim PET-CT would serve as a compass to optimize treatment. Also the role of PET in pre-transplant assessment is currently evolving. Much controversy surrounds the possibility of detecting relapse after completed treatment with the use of PET in surveillance in the absence of symptoms suggestive of recurrence and the results of published studies are rather discouraging because of low positive predictive value. This review presents current knowledge about the role of 18-FDG-PET/CT imaging at each point of management of patients with Hodgkin lymphoma.

Key words: positron emission tomog raphy, Hodgkin lymphoma, staging, interim PET, restaging.

Contemp Oncol (Pozn) 2017; 21 (2): 104-114 DOI: https://doi.org/10.5114/wo.2017.68618

\section{The role of FDG-PET in Hodgkin lymphoma}

\author{
Edyta Subocz ${ }^{1}$, Janusz Hałka1 ${ }^{1}$ Mirosław Dziuk ${ }^{2,3}$
}

${ }^{1}$ Department of Hematology, Military Institute of Medicine, Warsaw, Poland ${ }^{2}$ Affidea Mazovian PET/CT Center, Warsaw, Poland

${ }^{3}$ Nuclear Medicine Department, Military Institute of Medicine, Warsaw, Poland

\section{Introduction}

Over the past several years, the use of positron emission tomography (PET) in the management of many oncological diseases has significantly increased. It appears that 18-fluoro-2-deoxy-D-glucose positron emission tomography/computed tomography $\left({ }^{18} \mathrm{FDG}-\mathrm{PET} / \mathrm{CT}\right)$ is currently the most valuable technique for imaging changes in lymphomas [1, 2]. The Warburg phenomenon explains the possibility of imaging neoplasms with FDG-PET. In 1926, Otto Warburg discovered that cancer cells metabolize glucose via different processes in response to hypoxia. In brief, the anaerobic glucose cycle is energy inefficient; thus, cancer cells have an increased need for glucose, and trigger the mechanisms to fulfill it, such as increasing the amount of glucose transporting membrane proteins in the cell (GLUT1-5). Furthermore, tumor cells are characterized by high activity of hexokinase, an enzyme that catalyzes the first reaction of glucose metabolism. The resulting glucoso-6-phosphate, the first product of glycolysis, due to an inability to enter the process of aerobic glycolysis and hindered membrane transport, is "trapped" in the cancer cell. In PET, an analogue of glucose labeled with fluorine radioisotope (18-FDG) is used and undergoes the same changes as described above. Accumulation of 18-FDG-6-phosphate in tumor cells results in an increased emission of gamma quanta [3, 4]. This information, even in the case of a normal anatomical scan, reveals the functional pathologies of cells or tissues. The fusion of PET and CT images allows the precise location of these abnormalities to be determined [3]. It is important to note that fluorodeoxyglucose is not a specific marker for malignancy because it is also accumulated in the nonmalignant tissues that use glucose (such as the brain, brown fat, kidney, heart and activated inflammatory cells). However, this accumulation is usually less intense than in neoplasms and decreases with time due to the use of glucose in physiological processes and removing it from the cell. Paradoxically, in Hodgkin lymphoma, the increase in glucose uptake by inflammatory cells may be advantageous, because in the cancerous tissues, Reed-Sternberg and Hodgkin (RS and $\mathrm{H}$ ) cells constitute only $\sim 1 \%$ of all cells and are surrounded by non-cancerous mononuclear cells. Nonmalignant cells significantly enhance the PET-CT image through the accumulation of FDG, so they are visible in all patients with Hodgkin lympho$m a$ in the initial evaluation. A rapid decrease in metabolic activity of these cells during chemotherapy is the basis of PET use in the prediction of early response assessment $[5,6]$.

\section{PET in staging}

Accurate staging of Hodgkin lymphoma is very important because the extent of the disease process is a prognostic factor, and determines which treatment program will be followed. PET is used in planning of adjuvant radiotherapy (especially with new techniques), which translates into more precise target volume delineation for radiotherapy and thus protection of the healthy tissues $[6,7]$. The baseline PET-CT facilitates the subsequent 
evaluation tests, both in terms of the assessment of chemosensitivity and the effectiveness of completed treatment $[5,7]$. Since 1971, staging of lymphomas has been performed with the use of Ann Arbor classification, which, inter alia, was modified in 1988 at a conference in the Cotswolds, through the introduction of CT. Despite these early advances, we now know these methods are limited by the inability to evaluate organ function.

Many studies have shown that PET is both more accurate and more sensitive than $\mathrm{CT}$ in the initial staging of Hodgkin lymphoma. This conclusion is understandable considering the physiology of radioisotope tracer distribution and the mechanism of PET image formation presented briefly above [8-16]. FDG-PET/CT detects a higher number of lesions than conventional contrast-enhanced CT (CECT) in 25-30\% of patients [13-15]. Based on the results of previously published studies, it is difficult to specify the actual sensitivity of FDG-PET/CT in the staging of Hodgkin lymphoma due to the heterogeneous group of patients studied to date (including patients with $\mathrm{NHL}$ ) and the fact that FDG-PET has been performed both with or without CT $[11,14]$. The meta-analysis by Isasi et al. showed that the median sensitivity and specificity of FDG-PET in various lymphomas was $90 \%$ and $91 \%$, respectively and $93 \%$ and $88 \%$, respectively, in Hodgkin lymphoma [17]. In the study by Hutchings et al., which assessed only patients with Hodgkin lymphoma, the advantage of the sensitivity of FDG-PET and FDG-PET/CT versus CECT in primary staging was particularly pronounced in relation to extranodal changes $86 \%$ and $73 \%$ versus $37 \%$, respectively) and slightly less pronounced in the assessment of nodal involvement (92\% and 92\% versus $83 \%$, respectively) [14]. The possibility of imaging abnormal FDG uptake in normal-sized lymph nodes and organs with the correct structure as well as the lack of abnormal FDG uptake in the enlarged lymph nodes explain the greater sensitivity of PET compared with conventional CT [6].

When analyzing the results of studies conducted in groups of patients with Hodgkin lymphoma alone, it was evident that the use of PET or PET-CT was the reason for upstaging of the disease in $9-41 \%$ of patients and downstaging in $0-12 \%$ of patients, which translated into a change of treatment in 3-25\% of cases [8-16]. This analysis is presented in Table 1 . The upstaging was primarily associated with the detection of extranodal involvement (bone marrow, spleen, liver and lung) and to a lesser extent with the identification of lesions in additional lymph nodes (usually located below the diaphragm), which often appear as normal-sized in CT. The most common reason for the downstaging was due to the absence of abnormal uptake in the lymph nodes with a longitudinal dimension greater than $15 \mathrm{~mm}$; in $\mathrm{CT}$, this is often considered to be associated with the lymphoproliferative process [3, 6-16].

The most common cause of disease upstaging is the identification of bone marrow or bone involvement, which is confers stage IV disease affecting both treatment and prognosis (Fig. 1). Identifying affected bone marrow, as assessed by bone marrow trephine biopsy (BMB), is rare and occurs in $5 \%$ to $14 \%$ of cases. In patients in the early stages of the disease (IA-IIA), who have no additional adverse prognostic factors, this diagnostic rate drops to less than $1 \%[18-21]$.

The introduction of PET-CT has led to a significant improvement, with a 2-fold increase in diagnosed cases of bone marrow involvement in Hodgkin lymphoma, when compared to diagnosis by histopathology alone. The presence of multifocal FDG uptake in bone or bone marrow is sensitive for bone marrow involvement in Hodgkin lymphoma, even without the confirmed presence of RS-H cells via BMB. In some patients, PET shows diffusely increased uptake of fluorodeoxyglucose in the axial skeleton or in the long bones [22-25]. Although the meaning of this observation is still not entirely clear, it is believed to be a manifestation of bone marrow reactive hyperplasia induced by cytokines or associated anemia [22-26]. But you cannot say with certainty that diffused increased FDG uptake in the skeleton is non-neoplastic, as in individual patients in such cases, the bone marrow involvement was confirmed by BMB [16].

The sensitivity of FDG-PET/CT in the detection of bone marrow involvement is very high-close to $97 \%$ and the proportion of FDG-PET/CT-negative patients with a positive bone marrow biopsy among all cases is only 1.1\%. [27-29]. Based on these data, the new recommendations is that

Table 1. Studies comparing CT and PET for staging of Hodgkin lymphoma

\begin{tabular}{|c|c|c|c|c|c|}
\hline Author/year of publication & $\begin{array}{l}\text { Number of } \\
\text { patients }\end{array}$ & $\begin{array}{l}\text { FDG-PET or } \\
\text { FDG-PET/CT }\end{array}$ & $\begin{array}{c}\text { Upstaging } \\
\text { Number of patients (\%) }\end{array}$ & $\begin{array}{c}\text { Downstaging } \\
\text { Number of patients (\%) }\end{array}$ & $\begin{array}{c}\text { Treatment change } \\
\text { Number of patients (\%) }\end{array}$ \\
\hline Bangerter et al. 1998 [8] & 44 & FDG-PET & $5(12)$ & $1(2)$ & $6(14)$ \\
\hline Partridge et al. 2000 [9] & 44 & FDG-PET & $18(41)$ & $3(7)$ & $11(25)$ \\
\hline Jerusalem et al. 2001 [10] & 33 & FDG-PET & $3(9)$ & $4(12)$ & $1(3)$ \\
\hline Weihrauch et al. 2002 [11] & 22 & FDG-PET & $4(18)$ & $0(0)$ & $1(5)$ \\
\hline Munker et al. 2004 [12] & 73 & FDG-PET & $21(29)$ & $2(3)$ & nd \\
\hline Naumann et al. 2004 [13] & 88 & FDG-PET & $11(13)$ & $7(8)$ & $16(18)$ \\
\hline Hutchings et al. 2006 [14] & 99 & $\begin{array}{l}61 \text { out of } 99 \\
\text { FDG-PET/CT }\end{array}$ & 19 (19) & $5(5)$ & $9(9)$ \\
\hline Riggaci et al. 2007 [15] & 186 & FDG-PET & $27(14)$ & $3(1)$ & $11(6)$ \\
\hline Bednaruk-Mtyński et al. 2014 [16] & 96 & FDG-PET/CT & $27(28)$ & $6(6)$ & $20(21)$ \\
\hline
\end{tabular}



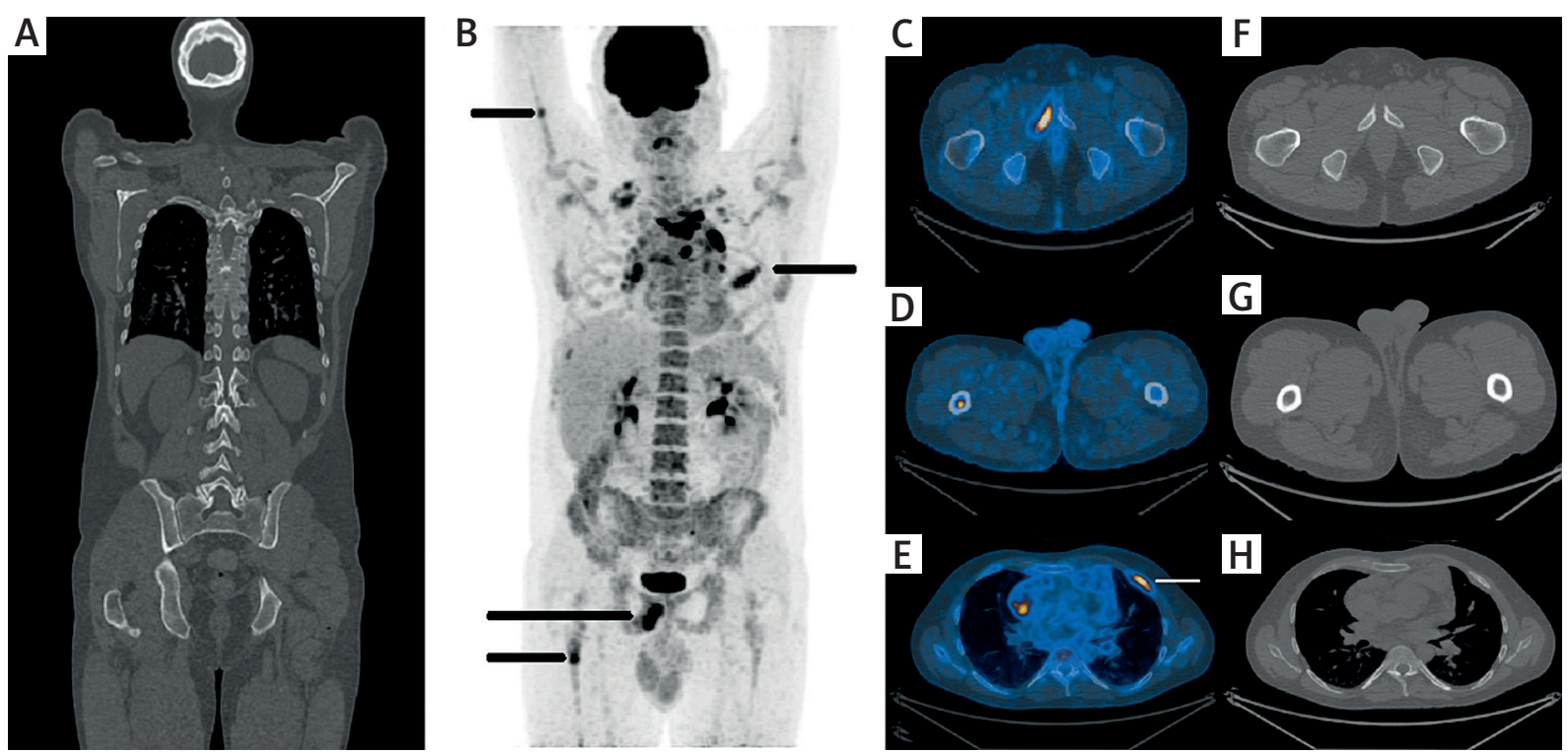

Fig. 1. CT (A) and PET (B) coronal images show a case of 35-y-old man with classical HL upstaged by PET-CT. Enlargement lymph nodes in mediastinum was reported on CT (stage 2). The PET scans showed multifocal uptake in bone marrow (stage 4). See arrows. Axial PET-CT images demonstrate lesions: in the right pubic bone (C), in the right femur (D) and in the left rib (E). Axial CT images in the same localizations show no irregularities (F, G, H)

a bone marrow biopsy is no longer needed for staging in patients with multifocal uptake in skeleton. In those cases with a PET-positive bone marrow finding, bone marrow biopsy should be performed only when the outcome could affect treatment decisions [3-4, 7].

There remains debate as to whether PET/CT alone is sufficient for the initial staging of Hodgkin disease. In this examination, lower-dose CT is performed to better localize abnormalities seen on PET and to correct for the attenuation of the radioactivity within the patient. In a number of studies conducted to date, unenhanced lower-dose FDG$\mathrm{PET} / \mathrm{CT}$ was confirmed to detect a larger number of lesions in comparison to contrast- enhanced CT (CECT) [30-32]. Performing PET and CECT in one examination (full-dose contrast-enhanced PET-CT) does not significantly affect the disease staging; however, the administration of intravenous contrast allows for better imaging of visceral organs and lymph nodes located below the diaphragm, particularly those lying in the vicinity of large vessels or intestines, which can be significant when planning radiotherapy $[4,5,7]$. Most often CECT is conducted before PET, and thus unenhanced lower-dose FDG-PET/CT is sufficient. If FDG-PET/CT is performed first, intravenous administration of contrast could be considered for patients who may require radiation therapy [1].

\section{PET in early response assessment}

Currently, the greatest interest of researchers in this field is the predictive value of interim PET-CT. Research efforts in this area are guided by the idea that PET performed early in the course of standard treatment could define two groups of patients: those who are chemosensitive and those who are chemorefractory. In the first group, this knowledge would allow for the prediction of cases when treatment could be less intense (shortening of chemother- apy and/or avoiding the adjuvant radiotherapy), which is important with respect to long-term complications such as secondary cancers or cardiovascular diseases. In the second case, positive PET would inform that the standard treatment is insufficient and should be intensified. In the case of a first-line treatment failure, prognosis is very poor, because only about $50 \%$ of patients can be cured by using salvage high-dose chemotherapy with autologous hematopoietic cells transplantation [33]. Therefore, early selection of chemoresistance seems to be essential in identifying patients for whom the standard ABVD treatment regimen (doxorubicin, bleomycin, vinblastine, dacarbazine) is insufficient. Of course, the opposite approach is also valid; specifically, the early administration of a more intense chemotherapy course for patients in the advanced stages of the disease, who have displayed increased risk factors, according to the BEACOPP scheme (bleomycin, etoposide, doxorubicin, cyclophosphamide, vincristine, procarbazine and prednisone) [33]. Although this intensified treatment plan is possible, it is often related to a higher risk of hematological toxicity, infectious complications, and the development of secondary cancers [34, 35]. In addition, chemotherapy according to the BEACOPP regimen, when compared to the ABVD regimen, results in significantly more cases of impaired fertility in both sexes [35]. This is particularly important given that the highest incidence of Hodgkin lymphoma is recorded in people 20-40 years of age [36].

Of all lymphomas, Hodgkin lymphoma is the one of most sensitive to treatment. The reduction in metabolic activity of RS and $\mathrm{H}$ cells, as well as cells from the microenvironment, acts as an "enhancer" of the PET image, and typically precedes the reduction in tumor volume, which usually takes some time. This phenomenon is called metabolic remission and is the basis for the early use of PET in 


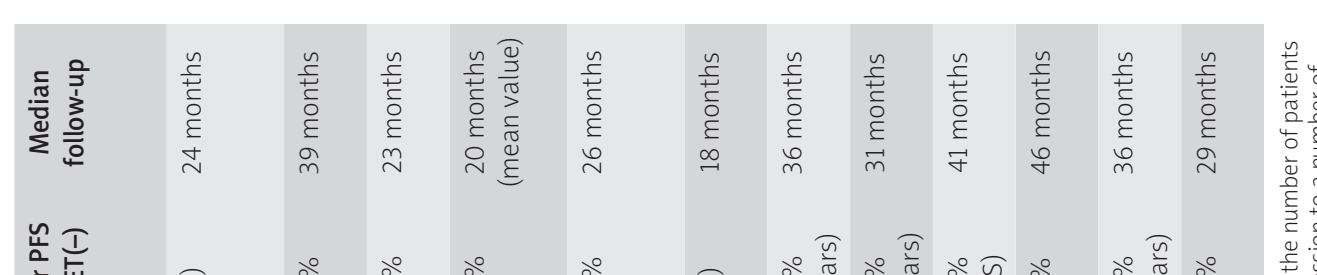

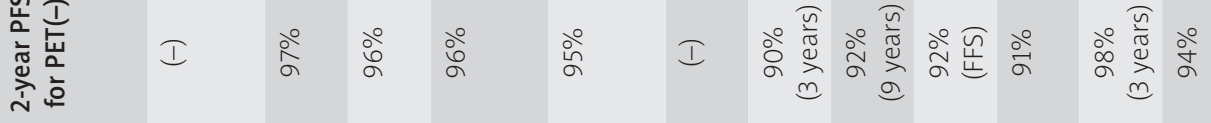

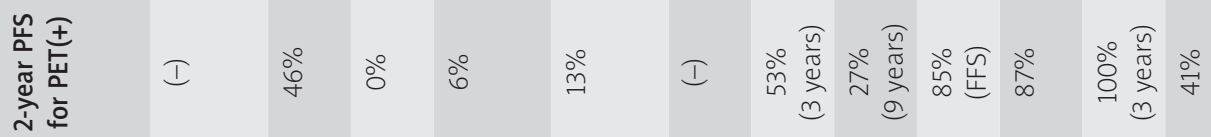

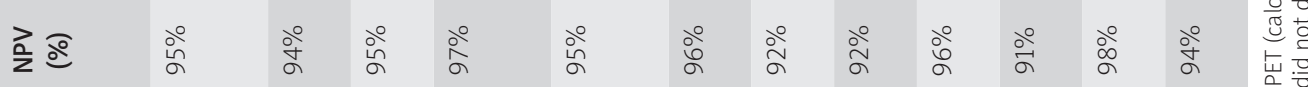

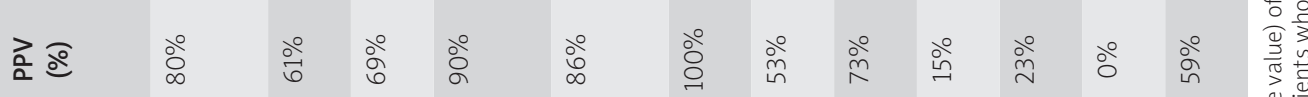

它套

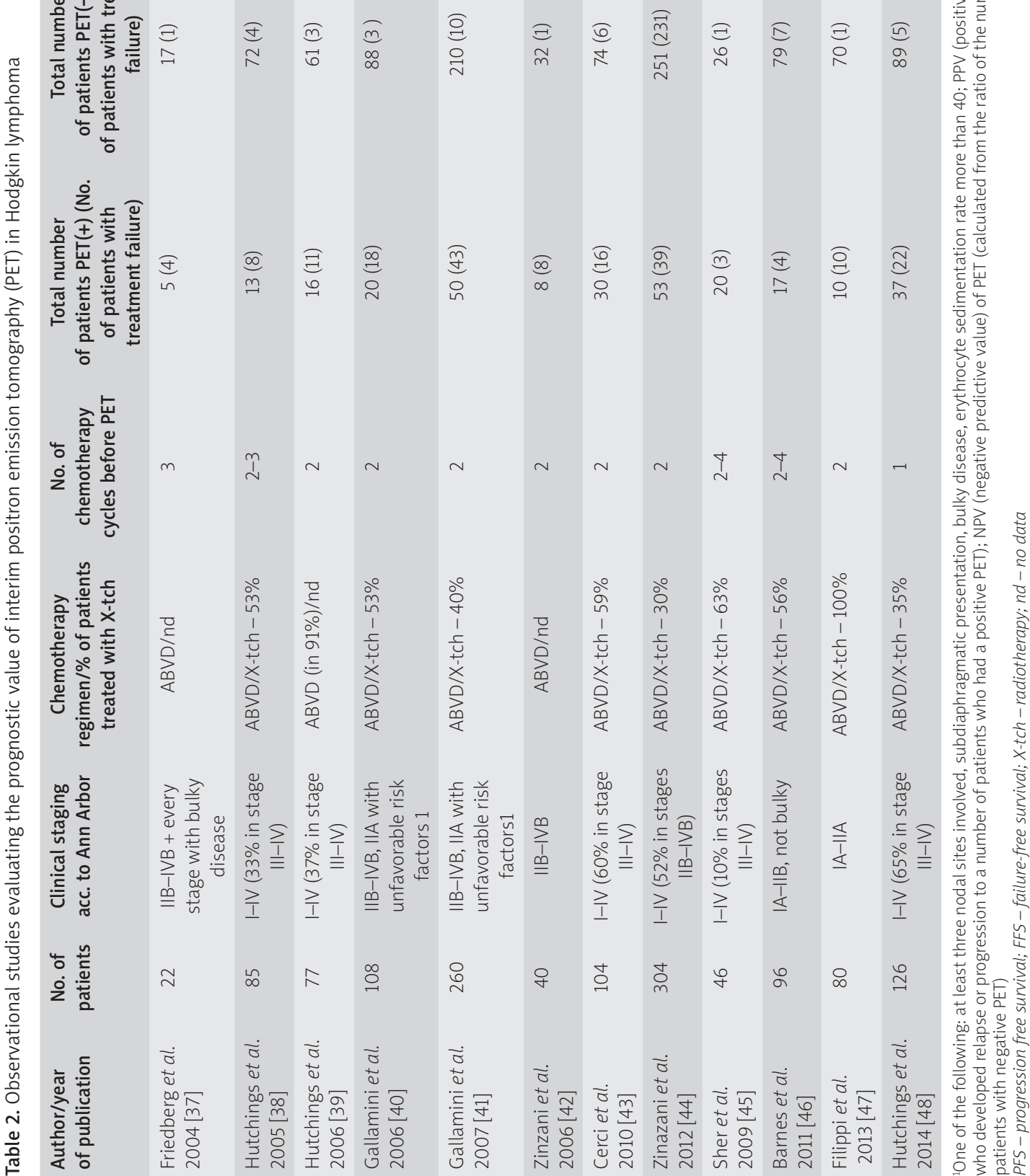


the evaluation of chemosensitivity [4, 6]. It is clear that the classical CT, which is based on anatomical criteria, gives way in this regard to functional imaging.

Most data on the prognostic value of PET comes from early studies of patients with advanced Hodgkin lymphoma treated with ABVD chemotherapy. Nearly $80 \%$ of these patients showed negative PET after two cycles of chemotherapy [37-48]; these results are summarized in Table 2. Hutchings et al. (2005) reported a significantly lower probability of a 2-year progression-free survival (PFS) in patients with positive PET after two or three cycles of ABVD chemotherapy compared to those with a negative result (46\% and $97 \%$, respectively) [38]. In the study by Gallamini et al., which included patients in advanced-stage Hodgkin disease, the difference was even greater: 13\% and 95\%, respectively. Importantly, Gallamini et al. also demonstrated the advantage of the predictive value of interim PET over the international prognostic score (IPS), which is commonly used to identify risk groups among patients with advanced disease [41]. In a 2009 meta-analysis of 360 patients with advanced Hodgkin lymphoma, after two to three cycles of ABVD chemotherapy, the sensitivity and specificity of interim PET was reported to be $81 \%$ and $97 \%$, respectively [49].

The results of subsequent studies of patients in early and intermediate stages of the disease, with no risk factors, showed that the positive predictive value (PPV) of interim PET and, consequently, the sensitivity of the examination are smaller [45-47] (Table 2). In the meta-analyses that followed, which included more than 1300 patients with Hodgkin lymphoma of all stages, the high negative predictive value (NPV) of early PET was confirmed, but sensitivity was moderate $(67-71 \%)[50,51]$. One of the reasons for this result was undoubtedly the fact that a larger part of the analyzed group consisted of patients in early stages of the disease, in which adjuvant radiotherapy of the areas primarily involved is standard practice. In the study by Filippi et al., which included patients with Hodgkin lymphoma at stages IA-IIA who were treated with combined chemoradiotherapy, none of the patients with positive early PET result were reported to have relapse of the disease during the 3-year follow-up [47].

In the observational studies analyzed above, in most cases PET was performed after two cycles of ABVD chemotherapy [37-47]. A recent study by Hutchings et al. showed a higher probability of a 2-year PFS in patients with negative PET performed after the first cycle of ABVD chemotherapy, when compared to positive PET ( $94 \%$ vs. $41 \%$, respectively). The PPV of PET after the first cycle of chemotherapy was moderate, at 59\%. In approximately half of the patients with positive PET after the first cycle of chemotherapy, the scans became negative after two cycles of treatment. Hutchings et al. suggest that a negative PET performed after the first cycle of chemotherapy could be a useful tool when making a decision as to whether to reduce the intensity of treatment [48]. Currently, no treatment modifications based on the results of interim PET are recommended [1, 2], however, such recommendations might be given, especially in the light of promising results of some recently published, multicenter PET-adapted clinical trials [52-56].

The two main lines of research attempting to use interim PET-CT are: firstly, the identification of a group of patients in whom it would be possible to safely reduce the intensity of treatment and thereby reducing the risk of related long-term complications; and, secondly the selection of patients for whom the standard therapy is suboptimal and should be reconsidered.

In a previous study by the National Cancer Institute of Canada Trials Group and the Eastern Cooperative Oncology Group, treatment with chemotherapy alone was compared to combined-modality therapy in patients with limited-stage Hodgkin lymphoma. The results, presented in 2005 with a median duration of follow-up of 4.2 years, indicated that freedom from disease progression in patients receiving radiotherapy was better than in patients treated with ABVD chemotherapy alone, but there were no differences between the groups in terms of overall survival (OS) [57]. This difference, however, was seen after a longer follow-up period (median: 11.3 years), when it became clear that the overall survival of patients treated with chemotherapy alone was higher than those with treatment that included radiotherapy (94\% vs. 87\%) [58]. The advantage of treatment with chemotherapy alone appeared to be due to fewer deaths from causes other than progressive Hodgkin lymphoma or acute treatment-related toxic effects. In the group of patients receiving radiotherapy, secondary cancers and cardiovascular complications were reported more frequently. It should be noted that in this study widefield radiotherapy was used, which was also the reason for a critical assessment of its results.

A multicenter EORTC/LYSA/FIL H10 trial investigated whether omission of involved-node radiotherapy affected PFS in patients in the early stages of Hodgkin lymphoma, who had negative PET-CT after two cycles of ABVD chemotherapy, and found a higher rate of early disease recurrence than in patients who received combination therapy (median follow-up: 1.1 years). However, it should be emphasized that the overall early outcome, both in the standard (combination therapy) and experimental (chemotherapy alone) arms of this study, was excellent. The aim of this trial was to find a reliable tool for the early identification of patients who could be spared the toxicity of radiotherapy, without compromising disease control. On the basis of statistical design, the experimental arm was unlikely to demonstrate noninferiority for chemotherapy alone; thus, this arm of the study was closed early for futility [52]. In the ultimate analysis published in 2017 in PET-negative patients, 5-year PFS rates in favourable (F) group were $99.0 \%$ vs. $87.1 \%$ in favor of combined modality treatment (CMT) and $92.1 \%$ versus $89.6 \%$ in unfavourable $(U)$ group, respectively. Fiveyear risk difference in PFS was $11.9 \%$ and $2.5 \%$ in favor of CMT in the $\mathrm{F}$ and $\mathrm{U}$ groups, respectively. The authors concluded, that the risk of relapse was higher in patients who were not treated with radiotherapy, especially in the F group [53].

However, it should be kept in mind that in the case of potentially curable cancers, such as Hodgkin lymphoma, the treatment itself can have a negative impact on pa- 
tient life expectancy. The question remains whether the benefits of slightly better early control of the disease, as achieved by combined-modality therapy, outweigh the potential risks of late complications of radiation therapy. If the answer is no, a novel question is raised: what degree of risk in worse early disease control is acceptable?

A recently published British RAPID trial assessed whether patients suffering from early-stage Hodgkin lymphoma (IA, IIA) with a negative PET-CT after three cycles of chemotherapy required adjuvant radiotherapy [54]. Accepting a limit of difference of no more than 7 percentage points with respect to the 3-year PFS, the authors found that the 3 -years PFS rate was $94.6 \%$ in the radiotherapy group and $90.8 \%$ in the group no further treatment. This result - an absolute risk difference of 3.8 percentage points - was within the range previously assumed by the authors, and it was concluded that the omission of adjuvant radiotherapy was not inferior to combination therapy in patients with nonbulky stages I-IIA Hodgkin lymphoma with negative PET-CT after three cycles ABVD chemotherapy with regard to PFS. It should be noted that a per-protocol analysis showed a higher 3-year PFS in the patients who received radiotherapy (97.1\%), with a rate ratio of 2.36 in favor of radiotherapy, when compared with the results of the aforementioned intention-to-treat analysis.

Both the RAPID trial and EORTC/LYSA/FIL H1O trial showed the use of adjuvant radiotherapy in patients in the early stages of Hodgkin lymphoma, who have negative PET scans, to be correlated with a slight improvement in PFS (3.8 percentage points in the ITT analysis and 6.3 percentage points in the per-protocol analysis in the RAPID trial). It seems that this effect may be disproportionate to the costs of exposure to radiation.

The use of interim PET for the early identification of patients who may benefit from the de-escalation of chemotherapy in advanced stages of Hodgkin lymphoma also appears to be an attractive approach. In 2016, the results of international, prospective, randomized RATHL trial were published. In this study, one of the research arms evaluated the safety of bleomycin removal from the ABVD regimen in patients in advanced stages of Hodgkin lymphoma, whose interim PET after two cycles of ABVD chemotherapy was negative. The results indicated that there was no significant difference with regard to 3-year PFS and OS in both groups (standard and experimental). The absolute difference in the 3-year PFS rate (ABVD minus AVD) was 1.6 percentage points. Median follow-up was 41 months. In addition, the omission of bleomycin following negative interim PET also reduced treatment-related pulmonary toxicity [55].

The conclusions from PET-adapted studies in groups of patients with advanced Hodgkin lymphoma, in which chemotherapy was escalated, based on positive interim PET results (NCRI RATHL, GITIL/FIL HD 0607, SWOG S0816), are also interesting. It turns out that chemotherapy according to a more aggressive treatment with BEACOPP regimen is effective in almost two-thirds of patients with a positive early PET. Three independent prospective studies showed that escalation to BEACOPP resulted in progression-free survival rates of 62 to $67 \%[55-56,59]$. These rates were essentially higher than the rates observed in retrospective studies in which patients continued ABVD (in 2-year analysis of 6 to $13 \%$ ) [40-41], and are consistent with the retrospective analysis of datasets of patients with advanced-stage Hodgkin lymphoma who switched to BEACOPP early [60]. Although the results of these studies strongly argues for a response-adapted approach with using interim PET-CT in advanced-stage Hodgkin lymphoma, we have to remember about an important limitation of such analyses. Namely, these analyses did not include a randomized comparison between continuation of standard ABVD chemotherapy and intensified BEACOPP, and their results are compared to the historic control group. Nevertheless, the concept of chemotherapy dose escalation based on positive interim PET is further supported by the results of EORTC/LYSA/FIL $\mathrm{H} 10$ study, in which patients with early stage disease who continued to have positive findings on interim PET-CT scan underwent. A significant improvement (13.2\%) of 5-year PFS was reached in the experimental arm (BEACOPP esC + INRTinvolved-node radiotherapy) compared with continuation of ABVD + INRT. In early PET-positive patients, 5-year PFS was $77.4 \%$ and $90.6 \%$ for standard ABVD + INRT arm and for BEACOPP esc +INRT arm, respectively [53].

Before the implementation of interim PET into a routine clinical practice as a kind of compass showing the approach that should be adopted in each patient would be possible, we need to obtain more convincing data from randomized clinical trials as well as to establish reliable methods to assess PET-CT scans. In 2009, at a conference in Deauville, a 5-point scale (5-PS) was introduced (shown in Table 3), which takes into account varying degrees of FDG uptake in tumor lesions as compared to uptake by reference organs such as the liver and mediastinal blood pool structure (MBPS) [61]. This scale replaced the previous dichotomous visual assessment, and divided changes into positive or negative, chose to use the mediastinum to define a negative scan in lesions $\geq 2 \mathrm{~cm}$ or adjacent background in smaller lesions. This scale was too restrictive in the context of the early assessment of chemosensitivity. In several retrospective studies, the usefulness of a 5 -point scale in the assessment of early PET in Hodgkin lymphoma was confirmed [62-64]. Moreover, in the aforementioned prospective, a randomized RATHL trial (designed to assess the role of an interim-adapted strategy in advanced Hodgkin lymphoma) found good agreement between local and expert readers in terms of assessment of response to treatment, as well as stage of disease, using a 5-point scale. The authors concluded that

Table 3. Deauville scale and IHP criteria

\begin{tabular}{|c|c|}
\hline Deauville 5-point scale (5-PS) & IHP criteria \\
\hline 1. No uptake & Positive PET: \\
\hline 2. Uptake $\leq$ MBPS & $\begin{array}{l}\text { Uptake }>\text { MBPS for } \\
\text { lesions }>2 \mathrm{~cm}\end{array}$ \\
\hline 3. Uptake > MBPS $\leq$ liver & $\begin{array}{l}\text { Uptake }>\text { background for } \\
\text { lesions }<2 \mathrm{~cm}\end{array}$ \\
\hline \multicolumn{2}{|l|}{$\begin{array}{l}\text { 4. Moderately increased uptake > } \\
\text { liver }\end{array}$} \\
\hline 5. Markedly increased uptake $>$ liver & \\
\hline
\end{tabular}


A

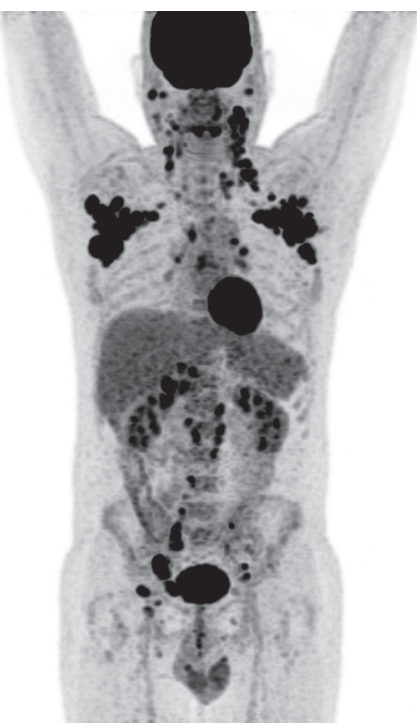

B

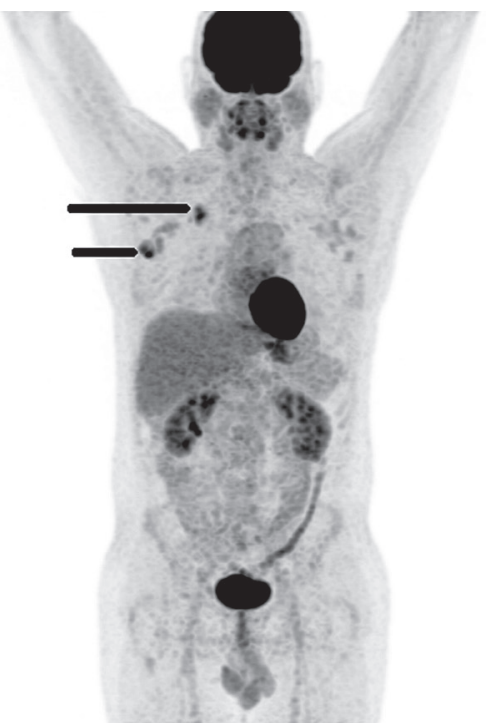

C

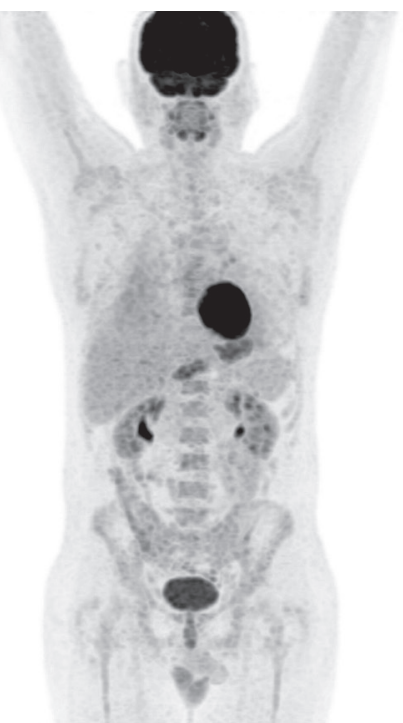

Fig. 2. Sequential PET images from patient with classical HL who achieved complete metabolic remission (CMR). A) Baseline PET of 47-y-old man with stage III. Patient initially had involvement of cervical and axillary regions, mediastinum and lymph nodes in abdomen and pelvis. B) Interim PET (after 2 cycles ABVD). Patient had persistent active disease in axillary lymph nodes (arrows), where FDG uptake was moderately higher than that in liver (score 4, positive according to 5PS). C) End-of-treatment PET (after 6 cycles ABVD). Lymph nodes showed FDG uptake below than that in mediastinum (score 2, negative according to 5PS)

A

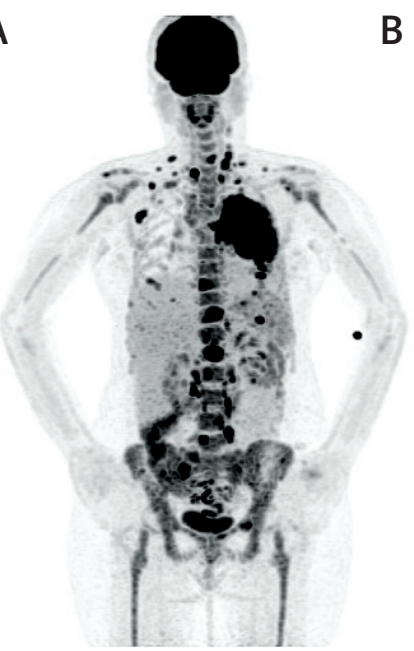

B

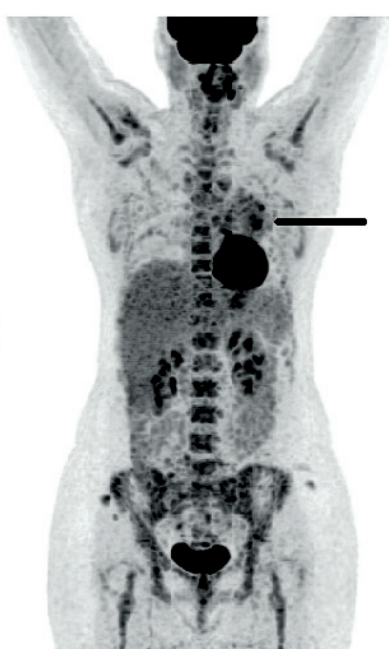

\section{C}

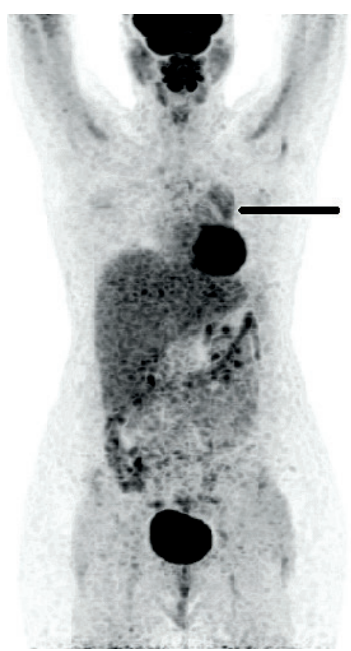

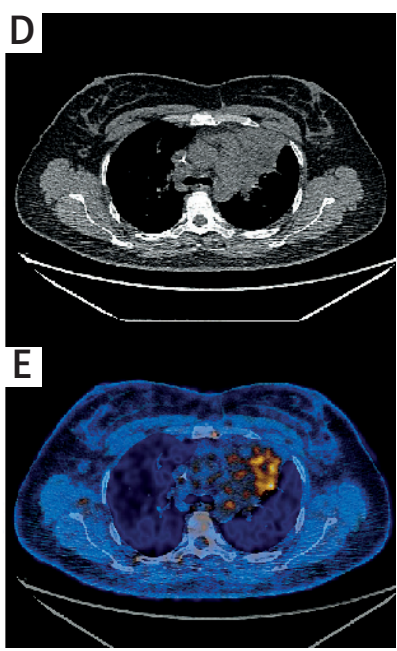

Fig. 3. Sequential PET images from patient with classical HL who not achieved CMR. A) Baseline PET of 36-y-old women with stage IV bulky. Patient initially had involvement of lymph nodes above and below diaphragm with bulky disease in mediastinum and abnormalities in lung, spleen, bone and bone marrow. B) Interim PET after 2 cycles ABVD, and C) End-of-treatment PET after 8 cycles ABVD: Patient had persistent active disease in residual mass in mediastinum (arrows). CT (D) and PET-CT (E) images indicate abnormal mass in traverse section. PET-CT demonstrated pathologic FDG uptake in the nodal mass, higher than that in liver (score 4, positive according to 5PS)

the 5-PS is the optimal method for response assessment in Hodgkin lymphoma when standardized PET protocols are used [65]. Figures 2 and 3 show two examples of sequential PET findings assessed using the Deauville 5-point scale.

\section{End-of-treatment PET}

The role of 18-FDG PET/CT in evaluating the effectiveness of first-line treatment is well documented, particularly for Hodgkin lymphoma, diffuse large B-cell lymphoma (DLBCL), and follicular lymphoma $[1,2,7]$. The value of PET performed following completion of treatment and its advantages over conventional $\mathrm{CT}$ in this setting have been demonstrated by several studies. The improved re- mission assessment using PET led to its incorporation in the recommendation for response assessment made by International Working Group (IWG) and published in 2007 [66]. This resulted in elimination of the unconfirmed complete remission $(\mathrm{CRu})$, and the introduction of the concept of metabolic complete remission (CMR). PET-CT is superior to other imaging methods because of its functional characteristics and its ability to distinguish between fibrotic tissue and residual viable disease. This is crucial in making subsequent treatment decisions, especially in the case of potentially curable lymphomas.

The advantage of PET has been demonstrated both in the case of ABVD and BEACOPP chemotherapy regimens 
A

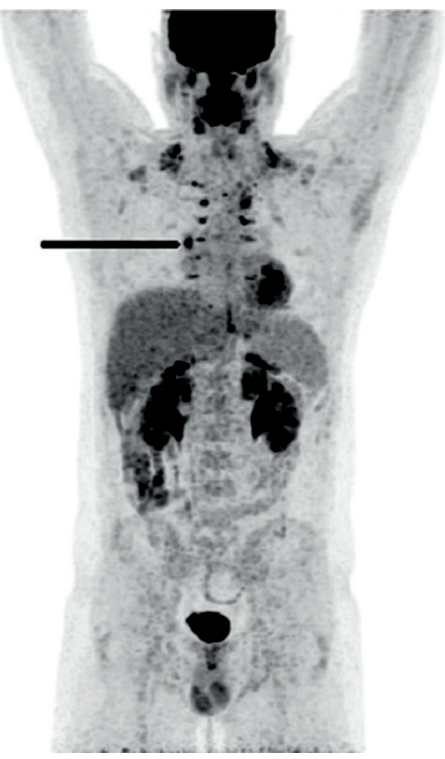

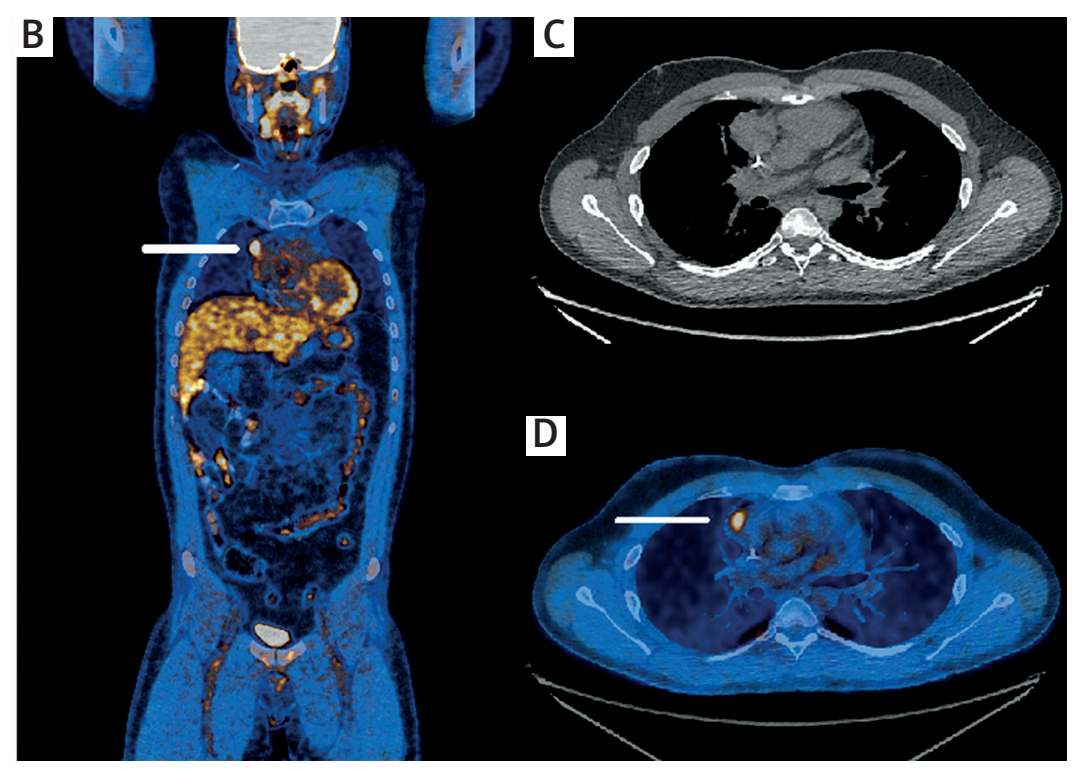

Fig. 4. PET (A), PET-CT (B) coronal images and axial CT (C) and PET-CT (D) scans show a case of 22-year-old man with stage IIA bulky classical HL who achieved CMR after combined-modality treatment. PET-CT was done in surveillance, 7 months after completed therapy, without any clinical suspicious of disease relapse and revealed pathologic FDG uptake (score 4 according 5PS) in residual mass in mediastinum (arrows). A biopsy was negative and patient remains free of disease

$[67,68]$. In a meta-analysis carried out by Zijlstra, pooled sensitivity and specificity of PET in the assessment of residual disease in Hodgkin lymphoma was observed to be $84 \%$ and $90 \%$, respectively [69]. The NPV end-of treatment PET is higher than the PPV due to the non-specific nature of FDG and possibly due to its accumulation in inflammatory tissues and in lesions with reactive changes after treatment. Therefore, it is important to maintain an adequate period between the end of treatment and the initiation of PET in order to reduce the likelihood of complicating the interpretation of PET scans. A minimum of 3 weeks after chemotherapy, and 12 weeks after the completion of radiotherapy, is typically recommended [66].

In the studies conducted to date, NPV of PET performed after completion of chemotherapy was rated at 94-100\%, and PPV at $91-92 \%$. Interestingly, when the study group consisted only of patients in the early stages of Hodgkin lymphoma, who had no unfavorable prognostic factors, PPV was only $46 \%[6,67,70]$. This suggests a higher percentage of false positive results in this group of patients. Therefore, if based on the positive results of PET salvage therapy is planned, consider a biopsy of residual metabolically active tissue, or if it is not possible, consider repeating PET in a reasonable time $[1,2]$.

Due to the fact that the predictive value of negative end-of-treatment PET is high and less reliable than PPV, it is believed that negative PET can be helpful in selecting patients in the advanced stages of the disease, for whom consolidating radiotherapy could be omitted. In the HD15 study, which included patients in stages IIB-IV treated with chemotherapy according to the BEACOPP regimen, PET was performed in a situation where in the end-of-treatment $C T$, residual changes greater than $2.5 \mathrm{~cm}$ were observed. Only those patients diagnosed with metabolic activity in residual changes underwent radiotherapy. Results of the treatment of patients who achieved partial remission according to the radiological criteria, but complete metabolic remission in PET, were similar to those patients who achieved complete radiological remission [68]. Despite the results of this study, the importance of metabolically-inactive residual changes after treatment, which do not meet the criteria of complete radiological remission, is not completely clear. Some studies suggest that a reduction of the dimension of less than $40 \%$, or the presence of residual masses greater than $4 \mathrm{~cm}$, imply a worse prognosis [71, 72].

Several studies also show that PET may be important in assessing the effectiveness of salvage chemotherapy before consolidating treatment with autologous hematopoietic stem cell transplantation [73-75]. Its predictive value exceeds assessment of remission by CT [76]. Current recommendations on the use and interpretation of PET in lymphoma stress that further clinical trials are necessary before PET can be recommended as a tool to help in the selection of candidates for high-dose chemotherapy with hematopoietic stem cell transplantation or for alternative treatments using new drugs [1].

\section{PET in surveillance}

Much controversy surrounds the possibility of detecting recurrence after completed first-line treatment with the use of PET-CT, guided by the idea of an effective treatment of recurrent cases in the case of early, asymptomatic imaging of relapse. To date, none of the recommendations, including NCCN (National Comprehensive Cancer Network), ESMO (European Society for Medical Oncology), or IWG, state that PET-CT should be routinely performed to monitor change after treatment $[1,2,36,77]$. The results of previous studies have not confirmed the significance of PET in this indication and they are discouraged because of low positive predictive value [78-80]. The false-positive 
rate with PET scans exceeding $20 \%$ and can lead to unnecessary further diagnostic procedures, exposure to ionizing radiation, patients anxiety and generates additional costs [2] (Fig. 4). Experts recommend performing PET only in case of clinical suspicion of relapse $[2,36,77]$.

The basic methods of control after treatment of lymphoma include careful history, physical examination and selected laboratory tests. In the case of Hodgkin lymphoma, which is potentially curable and risk of relapse decreases with time, the frequency of follow-up visits may be gradually reduced. In the first 2 years following remission, the follow-up examinations are recommended every 3-6 months, and every 6 to 12 months for a further 3 years. After this period, visits are recommended every year, primarily to monitor for late relapse and treatment-related adverse effects [36, 77].

\section{Summary}

The unique features of PET make it the most valuable imaging tool in the Hodgkin lymphoma. It is the most accurate method of assessing the extent of lymphoproliferative process at diagnosis, resulting in the selection of optimal treatment. Moreover, in some patients it is able to replace the histopathological analysis in the evaluation of bone and bone marrow involvement. Due to its functional character, PET is superior to classical CT in the assessment of remission after completed treatment; thus, PET/CT remains the gold standard in the two indications mentioned above. The possibility of personalizing treatment based on the results of early PET/CT also raises much hope for patients with this disease. The most convincing data, with respect to chemotherapy escalation on the basis of positive interim PET, are from patients in the advanced stages of the disease. However, due to the high number of false positives, PET is not entirely suited to control the disease after the completion of treatment and is not recommended for routine use in the absence of symptoms suggestive of recurrence.

Language support was provided by Proper Medical Writing, Warsaw, Poland.

The authors declare no conflict of interest.

\section{References}

1. Barrington SF, Mikhaeel NG, Kostakoglu L, et al. Role of imaging in the staging and response assessment of lymphoma: consensus of the International Conference on Malignant Lymphomas Imaging Working Group. J Clin Oncol 2014; 32: 3048-58.

2. Cheson BD, Fisher RI, Barrington SF, et al. Recommendations for initial evaluating, staging, and response assessment of Hodgkin and non-Hodgkin lymphoma: the Lugano classification. J Clin Oncol 2014; 32: 3059-68.

3. Cheson BD. Role of functional imaging in the management of lymphoma. J Clin Oncol 2011; 29: 1-11.

4. Gallamini A, Borra A. Role of PET in Lymphoma. Curr Treat Options Oncol 2014; 15: 248-61.

5. Gallamini A. Positron emission tomography scanning: a new paradigm for management of Hodgkin's lymphoma. Haematologica 2010; 95: 1046-8.
6. Barrington SF, Mikhaeel NG. When should DFG-PET be used in the modern management of lymphoma? Br J Haematol 2014; 164: 315-28

7. Kostakoglu L, Evans AM. FDG-PET imaging for Hodgkin lymphoma: current use and future applications. Clin Adv Haematol Oncol 2014; 12: 20-35.

8. Bangerter M, Moog F, Buchmann I, et al. Whole-body 2-[18F]-fluoro-2-deoxy-D-glucose positron emission tomography (FDG-PET) for accurate staging of Hodgkin's disease. Ann Oncol 1998; 9: 1117-22.

9. Partridge S, Timothy A, O'Doherty MJ, et al. 2-Fluorine-18-fluoro-2-deoxy-D glucose positron emission tomography in the pretreatment staging of Hodgkin's disease: Influence on patient management in a single institution. Ann Oncol 2000; 11: 1273-9.

10. Jerusalem G, Beguin Y, Fassotte MF, et al. Whole-body positron emission tomography using $18 \mathrm{~F}$-fluorodeoxyglucose compared to standard procedures for staging patients with Hodgkin's disease. Haematologica 2001; 86: 266-73.

11. Weihrauch MR, Re D, Bischoff S, et al. Whole-body positron emission tomography using 18F-fluorodeoxyglucose for initial staging of patients with Hodgkin's disease. Ann Hematol 2002; 81: 20-5.

12. Munker R, Glass J, Griffeth LK, et al. Contribution of PET imaging to the initial staging and prognosis of patients with Hodgkin's disease. Ann Oncol 2004; 15: 1699-704.

13. Naumann R, Beuthuen-Baumann B, Reiss A, et all. Substantial impact of FDG PET imaging on the therapy decision in patients with early-stage Hodgkin's lymphoma. Br J Cancer 2004; 90: 620-5.

14. Hutchings $M$, Loft A, Hansen $M$, et al. Positron emission tomography with or without computed tomography in the primary staging of Hodgkin's lymphoma. Haematologica 2006; 91: 482-9.

15. Rigacci L, Vitolo U, Nassi L, et al. Positron emission tomography in the staging of patients with Hodgkin's lymphoma: a prospective multicentric study by the Intergruppo Italiano Linfomi. Ann Hematol 2007; 86: 897-903

16. Bednaruk-Mlynski E, Pienkowska J, Skorzak A, et al. Comparison of positron emission tomography/computed tomography with classical contrast-enhanced computed tomography in the initial staging of Hodgkin lymphoma. Leuk Lymphoma 2015; 56: 377-82.

17. Isasi CR, Lu P, Blaufox MD. A metaanalysis of 18F-2-deoxy-2-fluoro-D-glucose positron emission tomography in the staging and restaging of patients with lymphoma. Cancer 2005; 104: 1066-74.

18. O'Carroll DI, McKenna RW, Brunning RD. Bone marrow manifestations of Hodgkin's disease. Cancer 1976; 38: 1717-28.

19. Munker R, Hasenclever D, Brosteanu O, et al. Bone marrow involvement in Hodgkin's disease: an analysis of 135 consecutive cases. German Hodgkin's Lymphoma Study Group. J Clin Oncol 1995; 13: 403-9.

20. Howell SJ, Grey M, Chang J, et al. The value of bone marrow examination in the staging of Hodgkin's lymphoma: a review of 955 cases in regional cancer centre. Br J Haematol 2002; 119: 408-11.

21. Vassilakopoulos TP, Angelopoulou MK, Constantinou N, et al. Development and Validation of a clinical prediction rule for bone marrow involvement in patients with Hodgkin lymphoma. Blood 2005; 105: 1875-80.

22. Pelosi E, Penna D, Douroukas A, et al. Bone marrow disease detection with FDG-PET/CT and bone marrow biopsy during the staging of malignant lymphoma: results from a large multicenter study. Q J Nucl Med Mol Imaging 2011; 55: 469-75.

23. Moulin-Romsee G, Hindié E, Cuenca X, et al. (18)3F-FDG PET/CT bone/bone marrow findings in Hodgkin's lymphoma may circumvent the use of bone marrow trephine biopsy at diagnosis staging. Eur J Nuc Med Mol Imaging 2010; 37: 1095-105.

24. Richardson S, Sudak J, Warbey V, et al. Routine bone marrow biopsy is not necessary in the staging of patients with classical Hodgkin lymphoma in the 18F-fluoro-2-deoxyglucose positron emission tomography era. Leuk Lymphoma 2012; 53: 381-5.

25. El-Galaly TC, d'Amore F, Mylam KJ, et al. Routine bone marrow biopsy has little or no therapeutic consequence for positron emission tomography/computed tomography-staged treatment-naïve patients with Hodgkin lymphoma. J Clin Oncol 2012; 30: 4508-14.

26. Salaun PY, Gastinne T, Bodet-Milian C, et al. Analysis of 18F-FDG PET diffuse bone marrow uptake and splenic uptake in staging of Hodgkin's lymphoma: a reflection of disease infiltration or just inflammation? Eur J Neucl Med Mol Imaging 2009; 36: 1813-21. 
27. Wu LM, Chen FY, Jiang XX, et al. 18F-FDG PET, combined FDG-PET/ $C T$ and $M R I$ for evaluation of bone marrow infiltration in staging of lymphoma: a systemic review and meta-analysis. Eur J Radiol 2012; 81: 303-11.

28. Adams HJ, Kwee TC, de Keizer B, et al. Systemic review and meta-analysis on the diagnostic performance of FDG-PET/CT in detecting bone marrow involvement in newly diagnosed Hodgkin lymphoma: is bone marrow biopsy still necessary? Ann Oncol 2014; 25: 921-7.

29. Pakos EE, Fotopoulos AD, Ioannidis JP. 18F-FDG PET for evaluation of bone marrow infiltration in staging of lymphoma: a meta-analysis. J Nucl Med 2005; 46: 958-63.

30. Schacher NG, Hanny TF, Taverna C, et al. Non-Hodgkin lymphoma and Hodgkin disease: coregistered FDG PET and CT at staging and restaging - do we need contrast-enhanced CT? Radiology 2004; 232: 823-9.

31. Raanani P, Shasha Y, Perry C, et al. Is CT scan still necessary for staging in Hodgkin and non-Hodgkin lymphoma patients in the PET/CT era? Ann Oncol 2006; 17: 117-22.

32. Elstrom R, Leonard JP, Coleman M, et al. Combined PET and lowdose, noncontrast CT scanning obviates the need for additional diagnostic contrast-enhanced CT scans in patients undergoing staging or restaging for lymphoma. Ann Oncol 2008; 19: 1770-3.

33. Canellos GP, Rosenberg SA, Friedberg JW, et al. Treatment of Hodgkin lymphoma: a 50-year perspective. J Clin Oncol 2014; 32: 163-8.

34. Diegl V, Franklin J, Pfreundschuh $M$, et al. Standard and increased-dose BEACOPP chemotherapy compared with COPP-ABVD for advanced Hodgkin's disease. N Engl J Med 2003; 348: 2386-95.

35. Cheson BD. Which Hodgkin's Patient in the Unites States Should Be Treated with BEACOPP? Curr Hematol Malig Rep 2014; 9: 222-6.

36. Eichenauer DA, Engert A, Ande M, et al. Hodgkin's lymphoma: ESMO Clinical Practise Guidelines for diagnosis, treatment and follow up. Ann Oncol 2014; 25: iii70-5.

37. Friedberg JW, Fischman A, Neuberg D, et al. FDG-PET is superior to gallium scintigraphy in staging and more sensitive in the follow-up of patients with de novo Hodgkin lymphoma: a blinded comparison. Leuk Lymphoma 2004; 45: 85-92.

38. Hutchings M, Mikhaeel NG, Fields PA, et al. Prognostic value of in terim FDG-PET after two or three cycles of chemotherapy in Hodgkin lymphoma. Ann Oncol 2005; 16: 1160-8.

39. Hutchings $M$, Loft $A$, Hansen $M$, et al. FDG-PET after two cycles of chemotherapy predicts treatment failure and progression-free survival in Hodgkin lymphoma. Blood 2006; 107: 52-9.

40. Gallamini A, Rigacci L, Merli F, et al. The predictive value of positron emission tomography scanning performed after two courses of standard therapy on treatment outcome in advanced stage Hod gkin's disease. Haematologica 2006; 91: 475-81.

41. Gallamini A, Hutchings M, Rigacci L, et al. Early interim 2-[18F]fluo ro-2-deoxy-D-glucose positron emission tomography is prognostically superior to international prognostic score in advanced-stage Hodgkin's lymphoma: a report front a joint Italian-Danish study. J Clin Oncol 2007; 25: 3746-52.

42. Zinzani PL, Tani M, Fanti S, et al. Early positron emission tomography (PET) restaging: a predictive final response in Hodgkin's disease patients. Ann Oncol 2006; 17: 1296-300.

43. Cerci JJ, Pracchia LF, Linardi CC, et al. 18F-FDG PET after 2 cycles of ABVD predicts event-free survival in early and advanced Hodgkin lymphoma. J Nucl Med 2010; 51: 1337-43.

44. Zinzani PL, Rigacci L, Stefoni V, et al. Early interim 18F-FDG PET in Hodgkin's lymphoma: evaluation on 304 patients. Eur J Nucl Med Mol Imaging 2012; 39: 4-12.

45. Sher DJ, Mauch PM, Van Den Abbeele A, et al. Prognostic significance of mid- and post-ABVD PET imaging in Hodgkin's lymphoma: the importance of involved-field radiotherapy. Ann Oncol 2009; 20: 1843-53.

46. Barnes JA, LaCasce AS, Zukotynski K, et al. End-of-treatment but not interim PET scan predicts outcome in nonbulky limited-stage Hodgkin's lymphoma. Ann Oncol 2011; 22: 910-5.

47. Filippi AR, Botticella A, Bellò M, et al. Interim positron emission tomography and clinical outcome in patients with early stage Hodgkin lymphoma treated with combined modality therapy. Leuk Lymphoma 2013; 54: 1183-7.
48. Hutchings M, Kostakoglu L, Zaucha JM, et al. In vivo sensitivity testing with positron emission tomography/computed tomography after one cycle of chemotherapy for Hodgkin lymphoma. J Clin Oncol 2014; 32: 2705-11.

49. Terasawa T, Lau J, Bardet S, et al. Fluorine-18-fluorodeoxyglucose positron emission tomography for interim response assessment of advanced-stage Hodgkin's lymphoma and difffuse large B-cell lymphoma: a systemic review. J Clin Oncol 2009; 27: 1906-14.

50. Ziakas PD, Poulou LS, Voulgarelis M, et al. The Gordian knot of interim 18-fluorodeoxyglucose positron emission tomography for Hodgkin lymphoma: a meta-analysis and commentary on published studies. Leuk Lymphoma 2012; 53: 2166-74.

51. Adams HJ, Nievelstein RA, Kwee TC. Prognostic value of interim FDG-PET in Hodgkin lymphoma: systematic review and meta-analysis. Br J Haematol 2015; 170: 356-66.

52. Raemaekers JM, André MP, Federico M, et al. Omitting radiotherapy in early positron emission tomography-negative stage I/II Hodgkin lymphoma is associated with an increased risk of early relapse: Clinical results of the preplanned interim analysis of the randomized EORTC/LYSA/FIL H10 trial. J Clin Oncol. 2014; 32: 1188-94.

53. André MP, Girinsky T, Federico M, et al. Early positron emission tomography response-adopted treatment in stage I and II Hodgkin lymphoma: Final results of the randomized EORTC/LYSA/FIL H10 trial. J Clin Oncol 2017; 35: 1786-94.

54. Radford J, Illidge T, Counsell N, et al. Results of a trial of PET-directed therapy for early-stage Hodgkin's lymphoma. N Engl J Med. 2015; 372: 1598-607.

55. Johnson P, Federico M, Kirkwood A, et al. Adapted treatment guided by interim PET-CT scan in advanced Hodgkin's lymphoma. N Engl J Med 2016; 374: 2419-29.

56. Press OW, Li H, Schöder H, et al. US intergroup trial of response-adapted therapy for stage III to IV Hodgkin lymphoma using early interim fluorodeoxyglucose-positron emission tomography imaging: Southwest Oncology Group S0816. J Clin Oncol 2016; 34: 2020-7.

57. Meyer RM, Gospodarowicz MK, Connors JM, et al. Randomized comparison of ABVD chemotherapy with a strategy that includes radiation therapy in patients with limited-stage Hodgkin's lymphoma: National Cancer Institute of Canada Clinical Trials Group and the Eastern Cooperative Oncology Group. J Clin Oncol. 2005; 23: 4634-42.

58. Meyer RM, Gospodarowicz MK, Connors JM, et al. ABVD alone versus radiation-based therapy in limited-stage Hodgkin's lymphoma. N Engl J Med 2012; 366: 399-408.

59. Gallamini A, Rossi A, Patti C, et al. Interim PET-adapted chemotherapy in advanced Hodgkin Lymphoma (HL). Results of the second interim analysis of the Italian GITL/FIL DH0607 Trial. Hematol Oncol (Oral Presentations) 2015; 33: 100-180; Abstract 118.

60. Gallamini A, Patti C, Viviani S, et al. Early chemotherapy intensification with BEACOPP in advanced-stage Hodgkin lymphoma patients with a interim-PET positive after two ABVD courses. $\mathrm{Br}$ J Haematol 2011; 152: 551-60.

61. Meignan M, Gallamini A, Hoioun C. Report on the First International Workshop on Interim-PET-Scan in Lymphoma. Leuk Lymphoma. 2009; 50: 1257-60.

62. Le Roux PY, Gastinne T, Le Gouill S, et al. Prognostic value of interim FDG PET/CT in Hodgkin's lymphoma patients treated with interim response-adapted strategy: comparison of International Harmonization Project (IHP), Gallamini and London criteria. Eur J Nucl Med Mol Imaging 2011; 38: 1064-71.

63. Biggi A, Gallamini A, Chauvie S, et al. International validation study for interim PET in ABVD-treated, advanced-stage Hodgkin lymphoma: interpretation criteria and concordance rate among reviewers. J Nucl Med 2013; 54: 683-90.

64. Gallamini A, Barrington SF, Biggi A, et al. The predictive role of interim positron emission tomography for Hodgkin lymphoma treatment outcome is confirmed using the interpretation criteria of the Deauville five-point scale. Haematologica 2014; 99: 1107-13.

65. Barrington SF, Kirkwood AA, Franceschetto A, et al. PET-CT for staging and early response: results from Response- Adapted Therapy in Advanced Hodgkin Lymphoma study. Blood 2016; 127: 1531-8. 
66. Juweid ME, Stroobants S, Hoekstra OS, et al. Use of positron emission tomography for response assessment of lymphoma: consensus of the Imaging Subcommittee of International Harmonization Project in Lymphoma. J Clin Oncol 2007; 25: 571-8.

67. Cerci JJ, Trindade E, Pracchia LF, et al. Cost effectiveness of positron emission tomography in patients with Hodgkin's lymphoma in unconfirmed complete remission or partial remission after firstline therapy. J Clin Oncol 2010; 28: 1415-21.

68. Engert A, Haverkamp H, Kobe C, et al. Reduced-intensity chemotherapy and PET-guided radiotherapy in patients with advanced stage Hodgkin's lymphoma (HD15 trial): a randomised, open-label, phase 3 non-inferiority trial. Lancet 2012; 379: 1791-9.

69. Zijlstra JM, Lindauer-van der Werf G, Hoekstra OS, et al. 18F-fluoro-deoxyglucose positron emission tomography for post-treat ment evaluation of malignant lymphoma: a systematic review. Haematologica 2006; 91: 522-9.

70. Kobe C, Dietlein M, Franklin J, et al. Positron emission tomogra phy has a high negative predictive value for progression or early relapse for patients with residual disease after first-line chemotherapy in advanced-stage Hodgkin lymphoma. Blood 2008; 112: 3989-94.

71. Kobe C, Kuhnert G, Kahraman D, et al. Assessment of tumor size reduction improves outcome prediction of positron emission tomography/computed tomography after chemotherapy in advanced-stage Hodgkin lymphoma. J Clin Oncol 2014; 32: 1776-81.

72. Magagnoli M, Marzo K, Balzarotti M, et al. Dimension of residual CT scan mass in Hodgkin's lymphoma $(\mathrm{HL})$ is a negative prognostic factor in patients with PET negative after chemo +/- radiotherapy. Blood 2011; 118: Abstract 93.

73. Jabbour E, Hosing C, Ayers G, et al. Pretransplant positive positron emission tomography/gallium scans predict poor outcome in patients with recurrent/refractory Hodgkin lymphoma. Cancer 2007; 109: 2481-9.

74. Moskowitz AJ, Yahalom J, Kewalramani T, et al. Pretransplantation functional imaging predicts outcome following autologous stem cell transplantation for relapsed and refractory Hodgkin lymphoma. Blood 2010; 116: 4934-7.

75. Smeltzer JP, Cashen AF, Zhang Q, et al. Prognostic significance of FDG-PET in relapsed or refractory classical Hodgkin lymphoma treated with standard salvage chemotherapy and autologous stem cell transplantation. Biol Blood Marrow Transplant 2011; 17: 1646-52.

76. Terasawa T, Dahabreh IJ, Nihashi T. Fluorine-18-fluorodeoxyglucose positron emission tomography in response assessment before high-dose chemotherapy for lymphoma: a systematic review and meta-analysis. Oncologist 2010; 15: 750-9.

77. Hoppe RT, Advani RH, Ai WZ, et al. Hodgkin lymphoma, version 2.2015. J Natl Compr Canc Netw 2015; 13: 554-86.

78. Jerusalem G, Beguin Y, Fassotte MF, et al. Early detection of relapse by whole-body positron emission tomography in the follow-up of patients with Hodgkin's disease. Ann Oncol 2003; 14: 123-30.

79. El-Galaly TC, Mylam KJ, Brown P, et al. Positron emission tomography/computed tomography surveillance in patients with Hodgkin lymphoma in first remission has a low positive predictive value and high costs. Haematologica 2012; 97: 931-6.

80. Gandikota N, Hartridge-Lambert S, Migliacci JC, et al. Very low utility of surveillance imaging in early-stage classic Hodgkin lymphoma treated with a combination of doxorubicin, bleomycin, vinblastine, and dacarbazine and radiation therapy. Cancer 2015; 121: $1985-92$

\section{Address for correspondence}

\section{Edyta Subocz}

Department of Hematology

Military Institute of Medicine

Szaserow 128

04-141 Warsaw, Poland

e-mail: Suboczka@poczta.onet.pl

Submitted: 9.12.2015

Accepted: 20.03 .2016 